\title{
Hyperintense Dentate Nuclei on T1-Weighted MRI: Relation to Repeat Gadolinium Administration
}

\author{
M.E. Adin, L. Kleinberg, D. Vaidya, E. Zan, (DS. Mirbagheri, and IDD.M. Yousem
}

\begin{abstract}
BACKGROUND AND PURPOSE: A hyperintense appearance of the dentate nucleus on T1-weighted MR images has been related to various clinical conditions, but the etiology remains indeterminate. We aimed to investigate the possible associations between a hyperintense appearance of the dentate nucleus on Tl-weighted MR images in patients exposed to radiation and factors including, but not limited to, the cumulative number of contrast-enhanced MR images, amount of gadolinium administration, dosage of ionizing radiation, and patient demographics.
\end{abstract}

MATERIALS AND METHODS: The medical records of 706 consecutive patients who were treated with brain irradiation at The Johns Hopkins Medical Institutions between 1995 and 2010 were blindly reviewed by 2 readers.

RESULTS: One hundred eighty-four subjects were included for dentate nuclei analysis. Among the 184 subjects who cumulatively underwent 2677 MR imaging studies following intravenous gadolinium administration, 103 patients had hyperintense dentate nuclei on precontrast T1-weighted MR images. The average number of gadolinium-enhanced MR imaging studies performed in the group with normal dentate nuclei was significantly lower than that of the group with hyperintense dentate nuclei. The average follow-up time was 62.5 months. No significant difference was observed between hyperintense and normal dentate nuclei groups in terms of exposed radiation dose, serum creatinine and calcium/phosphate levels, patient demographics, history of chemotherapy, and strength of the scanner. No dentate nuclei abnormalities were found on the corresponding CT scans of patients with hyperintense dentate nuclei $(n=44)$. No dentate nuclei abnormalities were found in 53 healthy volunteers.

CONCLUSIONS: Repeat performance of gadolinium-enhanced studies likely contributes to a long-standing hyperintense appearance of dentate nuclei on precontrast T1-weighted-MR images.

ABBREVIATIONS: CEMRI = contrast-enhanced MRI; DN = dentate nucleus; $\mathrm{HDN}=$ hyperintense appearance of the dentate nucleus; $\mathrm{NDN}=$ normal dentate nucleus; MFS $=$ magnetic field strength of the scanner; $\mathrm{p} 25=25$ th percentile; $\mathrm{p} 75=75$ th percentile; $\mathrm{RD}=$ radiation dose; $\mathrm{RT}=$ radiation therapy

A hyperintense appearance of the dentate nucleus (HDN) on precontrast T1-weighted MR imaging has been reported to be related to various clinical conditions. There is no consensus about the underlying etiology, and the mechanism and clinical implications remain poorly understood. A number of variables may have direct or indirect effect on the dentate nucleus (DN). Among those factors, radiation therapy (RT) has well-known short-term and long-term effects on the CNS. ${ }^{1} \mathrm{RT}$ is reported to

Received December 20, 2014; accepted after revision February 19, 2015.

From the Division of Neuroradiology (M.E.A., E.Z., S.M., D.M.Y.), The Russell H. Morgan Department of Radiology and Radiological Science; Department of Radiation Oncology and Radiation Molecular Sciences (L.K.); and Johns Hopkins Bloomberg School of Public Health (D.V.), The Johns Hopkins Medical Institutions, Baltimore, Maryland.

Please address correspondence to Mehmet Emin Adin, MD, Johns Hopkins Medical Institutions, 600 N. Wolfe St. Phipps B100, Baltimore, MD 21287; e-mail: emin.adin@gmail.com

http://dx.doi.org/10.3174/ajnr.A4378 cause calcification in brain tissue. ${ }^{2,3}$ A retrospective study conducted on pediatric patients suggested that the DN is particularly sensitive to the effects of brain irradiation, leading to structural changes on DTI. ${ }^{4}$

Few studies in the English literature have investigated HDN on T1WI. ${ }^{5-8}$ The possible etiologies of HDN remain indeterminate. The appearance has been attributed to radiation therapy, the secondary-progressive subtype of MS, and, most recently, to cumulative numbers of gadolinium-enhanced MR imaging scans. ${ }^{5,6}$ No long-term longitudinal radiologic studies have been completed defining the radiologic course of HDN, to our knowledge. The frequency of HDN in the healthy population has not been determined because none of the existing studies included a healthy control group to provide a comparison.

The purpose of our study was to explore associations of HDN in irradiated patients with long-term follow-up MRI studies. We examined various factors including the cumulative number of 
gadolinium-enhanced MR images, total amount of gadolinium administration, different commercial gadolinium agents, dosage of therapeutic ionizing radiation, histology of underlying brain neoplasm, calcium/phosphate level, creatinine levels, magnetic field strength of the scanner (MFS), patient demographics, and chemotherapy exposure, by using a large cohort with retrospective evaluation of long-term longitudinal follow-up brain MR imaging studies.

\section{MATERIALS AND METHODS}

We retrospectively reviewed the medical records of 706 consecutive patients who were treated with brain irradiation for primary brain neoplasms at The Johns Hopkins Medical Institutions between June 1995 and January 2010. The institutional review board approved our study in compliance with the Health Insurance Portability and Accountability Act and waived informed consent. We reviewed clinical notes, discharge summaries, operative notes, radiologic studies, RT summaries, and prior studies performed at outside institutions, when available. In a subset group, we investigated whether there was a threshold value of gadolinium administration before HDN is evident and whether there is a significant difference among gadolinium agents used during the follow-up. We excluded all cases that lacked the exact dosage and gadolinium agent information. In this group, for the same reason, we excluded cases with outside studies for which such information was not available.

Radiologic interpretations for all available MR images for all subjects were made independently by 2 radiologists who were blinded to clinical diagnoses and treatment protocols. A determination of HDN was made on the basis of the qualitative comparison of the signal intensity of the middle cerebellar peduncle versus the DN on T1WI, because white matter tracts (middle cerebellar peduncle) are typically brighter than gray matter nuclei in the normal state on T1WI. Once HDN was determined for a subject, comparative assessment of prior studies was done to detect the first appearance of HDN, and follow-up studies were reviewed to look for its disappearance with time. Any disagreement between readers regarding final conclusions was resolved by discussion and consensus. The senior author (D.M.Y.), who has 25 years' experience, was used as the tiebreaker when there were ambiguities as to the signal intensity of the DN. No $\kappa$ tests were performed because there were only 9 scans of all MR imaging studies that required such a review for signal intensity. CT scans of patients demonstrating HDN were also reviewed by using the same methodology $(n=54)$. CT studies with severe artifactual degradation in the posterior fossa and those that were performed only after iodine enhancement were excluded $(n=10)$.

The MFS was noted for each study to investigate its influence on the bright appearance of the DN. RT was classified according to site, dose, and number of fractions applied in each individual. Approximate radiation exposure to the DN for each subject was quantitatively calculated and depicted by color isodose lines applied to multiplanar CT and MR imaging of each individual's RT therapy plan. The treatment plans were created by using the Pinnacle $^{3}$ Planning system (Philips Healthcare, Best, the Netherlands), Leksell GammaPlan (Elekta Instruments, Stockholm, Sweden), Brainlab (Brainlab, Feldkirchen, Germany), or Multi- plan (Accuray, Sunnyvale, California). The radiation dose (RD) and techniques were specific to the patients' illnesses and included stereotactic single treatment or hypofractionated radiosurgery plans for acoustic neuroma or meningioma of 1200-1600 cGy in a single fraction or $2500 \mathrm{cGy}$ hypofractionated for 5 days, delivered with a dose distribution tightly conforming to the abnormality. Patients with meningiomas or gliomas were treated to wider areas of normal-appearing brain, and for larger or infiltrating tumors, 4500-6000 cGy was delivered during 25-33 treatment days by $3 \mathrm{D}$ and intensity-modulated treatment approaches. The radiation treatment plan, containing isodose lines pictorially demonstrating the radiation dose distributed throughout the brain, was used to determine a quantitative value of the dose delivered to the DN. In general, there was not specific quantification or depiction of the $\mathrm{RD}$ for areas of the brain receiving $<20 \%$ of the dose prescribed to the tumor, and this region was considered outside the radiated brain. If the $\mathrm{DN}$ was out of the region of the brain receiving $>20 \%$ of the dose targeted at the tumor, an approximate value according to the total $\mathrm{RD}$ was recorded. These values were accepted as negligible in amplitude and were censored in statistical analyses.

Underlying brain tumors were classified according to their histologic type. Patient demographics and serum calcium phosphate levels at the time of detection of HDN were also detailed. Chemotherapy regimen, if any, was also investigated.

We excluded the following: patients with no MR images available in the radiology archive $(n=458)$, MR imaging studies degraded with severe artifacts, a surgical cavity, or any type of posterior fossa lesion that obscured thorough examination of the DN ( $n=23 ; 21$ adult and 2 pediatric cases), patients with hepatic dysfunction $(n=1)$, patients with a history of total parenteral nutrition and/or manganese intake $(n=3)$, and patients who did not have MR imaging available after brain irradiation $(n=37)$. All studies were performed by using either 1.5T (Magnetom Avanto and Magnetom Espree; Siemens, Erlangen, Germany; Intera and Achieva; Philips Healthcare; and Signa; GE Healthcare, Milwaukee, Wisconsin) or 3T (Verio and Skyra; Siemens; and Achieva; Philips Healthcare) MR imaging machines. The T1WI used one of the following: 1) an MPRAGE pulse sequence with $8^{\circ}-9^{\circ}$ flip angle and TR, $1900-2200 \mathrm{~ms}$; TE, 2-5 ms; 2) a spin-echo pulse sequence with TR, 400-600 ms; TE, 2-20 ms; or 3) a T1weighted FLAIR sequence with TR, $2000 \mathrm{~ms}$; TI, $860 \mathrm{~ms}$; and TE, $8-12 \mathrm{~ms}$. No specific methodology was used to enroll subjects for either $1.5 \mathrm{~T}$ or $3 \mathrm{~T}$ scanners; however, the number of $1.5 \mathrm{~T}$ studies exceeded that of $3 \mathrm{~T}$ scans because the institution did not purchase $3 \mathrm{~T}$ scanners until the most recent 10 years and the study spanned 1995-2010. In our institution, and thus in the present study, we used almost exclusively Magnevist (gadopentetate dimeglumine; Bayer HealthCare Pharmaceuticals, Wayne, New Jersey) for contrast-enhanced MR imaging (CEMRI) in subjects with normal renal function and without known Magnevist contrast reactions. Omniscan (gadodiamide; GE Healthcare, Piscataway, New Jersey), MultiHance (gadobenate dimeglumine; Bracco Diagnostics, Princeton, New Jersey), ProHance (gadoteridol; Bracco Diagnostics), OptiMARK (gadoversetamide; Mallinckrodt, St. Louis, Missouri), and Gadavist (gadobutrol; Bayer Schering Pharma, Berlin, Germany) were other agents used in a small proportion of the 
CEMRI scans. In 113 cases, all individual consecutive studies were performed by using Magnevist (gadopentetate dimeglumine). In most of the remaining cases, different combinations of gadolinium molecules were injected with an unevenly distributed dosage. The renal function of subjects was evaluated by using the most recent serum creatinine levels at the time of the relevant MR imaging scan, and the estimated glomerular filtration rate was calculated when possible.

To investigate the incidence of HDN on T1WI in the general population and justify the qualitative methodology, we recruited a control group consisting of 53 healthy volunteers (age range, 22-75; mean age, $41.9 \pm 11.8$ years; 15 women and 38 men). None of the control group participants underwent prior CEMRI scanning of any body part. The total number of MR imaging scans in the control group was 133 (mean, $2.5 \pm 2.3$ ). MPRAGE (either 1.5T Magnetom Avanto and Magnetom Espree [Siemens] or 3T Verio and Skyra [Siemens]) was used in evaluating the DN in 52 control subjects, and 3D fast echo-spoiled gradient echo (1.5T Signa; GE Healthcare) was used in 1 control subject. We qualitatively compared the signal intensities of the DN and middle cerebellar peduncle by using ROI measurement in this group. None of the control group participants underwent cranial CT imaging.

For statistical analyses, demographic, clinical, laboratory, and outcome measures were tabulated in 2 groups: those with normal

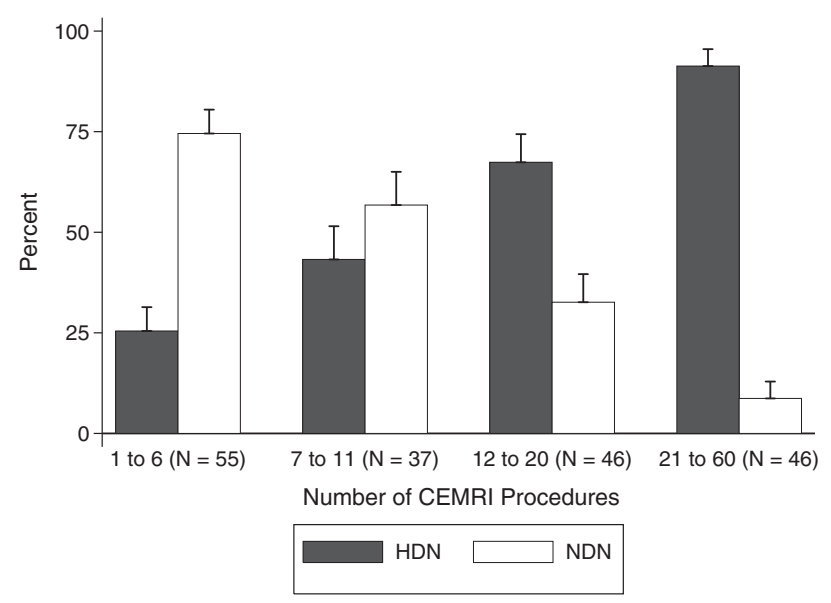

FIG 1. Graph shows the number of contrast-enhanced MR imaging scans divided into 4 subgroups for both the HDN and NDN groups. The likelihood of developing HDN significantly increases in those with a history of $>6$ CEMRI scans.

Table 1: Demographics and diagnoses of the pediatric age group tabulated as NDN versus HDN $^{\mathrm{a}}$

\begin{tabular}{lcc}
\hline \multicolumn{1}{c}{ Variable/Group } & NDN ( $\boldsymbol{n}=\mathbf{5})(\%)$ & HDN $(\boldsymbol{n}=\mathbf{1 5})(\%)$ \\
\hline Race (white versus nonwhite) & $3 \mathrm{~W}(100)$ & $11 \mathrm{~W}(73), 4 \mathrm{NW}(27)$ \\
Sex (female vs male) & $3 \mathrm{~F}(60), 2 \mathrm{M}(40)$ & $5 \mathrm{~F}(33), 10 \mathrm{M}(67)$ \\
Total RT dose mean (SD) (cGy) & $5628(222)$ & $5196(680)$ \\
Anaplastic astrocytoma & $1(20.0)$ & $1(6.7)$ \\
Astrocytoma & $0(0.0)$ & $3(20.0)$ \\
Astrocytoma, pilocytic & $1(20.0)$ & $2(13.3)$ \\
Desmoplastic nodular medulloblastoma & $0(0.0)$ & $1(6.7)$ \\
Ependymoma & $1(20.0)$ & $2(13.3)$ \\
Glioma, malignant & $1(20.0)$ & $0(0.0)$ \\
Medulloblastoma & $1(20.0)$ & $5(33.3)$ \\
Primitive neuroectodermal tumor & $0(0.0)$ & $1(6.7)$ \\
\hline
\end{tabular}

Note:-W indicates white; NW, nonwhite.

${ }^{a}$ Note a significantly higher HDN percentage compared with the adult population. dentate nuclei (NDN) and those with HDN. Categoric variables were tabulated as number (percentage within group), and continuous variables were tabulated as median (25th percentile [p25] to 75 th percentile[p75]). Group differences were tested by using $\chi^{2}$ or Fisher exact tests (if cell counts were low) for categoric variables and nonparametric rank sum tests for continuous variables. The variable specific dose was left-censored at $500 \mathrm{U}$ when the $\mathrm{DN}$ was located beyond the outer dose lines representing the lowest amount of radiation, generally $<20 \%$ of the $\mathrm{RD}$ prescribed to the tumor, which is considered to have a minimal risk of causing toxicity or tissue injury/side effects. Rank sum statistics were conservative for this variable because the median in both groups lay in the censored range. We plotted the percentage of individuals with HDN versus quartiles of the CEMRI number as a bar chart along with their 95\% confidence intervals (Fig 1). We performed logistic regression analyses for HDN versus NDN as the dependent variable with the number of CEMRIs as the independent variable adjusted for age, sex, and race (classified as white versus nonwhite). The variable "total number of CEMRI" was highly skewed and not normally distributed. Because a few individuals received high numbers of CEMRIs, we logarithmically transformed this variable to limit the undue influence of these high numeric values on the regression results.

\section{RESULTS}

We evaluated 184 subjects in this study ( 83 females, 101 males). The ages of subjects ranged from 3 to 73 years (mean, $43.3 \pm 16.8$ years). Twenty subjects were younger than 18 years of age (Table 1). Among the 184 subjects who altogether underwent $2677 \mathrm{MR}$ imaging studies (mean, 14.55; range, 1-60) following IV gadolinium administration, 103 (55.9\%) patients showed HDN on T1WI. The estimated glomerular filtration rate was measured in 84 subjects and was $60 \mathrm{~mL} / \mathrm{min}$ per $1.73 \mathrm{~m}^{2}$ in all subjects. Of these, 25 had NDN, and 59, HDN. The upper normal limit of the creatinine value was accepted as $1.1 \mathrm{mg} / \mathrm{dL}$. In only 3 cases was the creatinine value $>1.1 \mathrm{mg} / \mathrm{dL}$ (range, $0.2-1.4 \mathrm{mg} / \mathrm{dL}$ ). The median (p25 to p75) serum creatinine value was $0.7(0.5-0.8)$ for NDN (56 individuals) and 0.65 (0.-0.8) for HDN (92 individuals). The rank sum test $P$ value for the difference between groups was .99 .

\section{Strong Impact of the Number of CEMRIs and Volume of Gadolinium Administered}

The average number of CEMRIs for the NDN group was significantly lower than that of HDN group (median [p25 to p75] for NDN, 6 [4.0-11.0]; range, $1-25$; and median [p25 to p75] for HDN, 18 [10.0-26.0]; range, 2-60; $P<$ $.001)$. The logistic regression model was used to assess the impact of factors that may relate to HDN on T1WI. A strong association was found between the total number of contrast-enhanced MR imaging studies and the existence of HDN on T1WI. After we adjusted for age, sex, nonwhite race, and follow-up time in logistic regression, doubling the number 

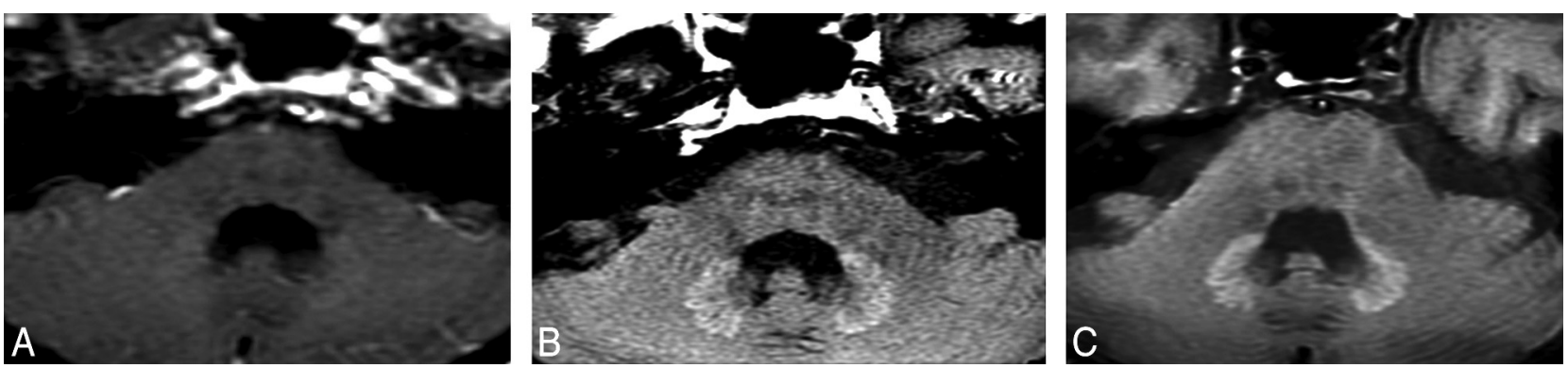

FIG 2. HDN on axial TIWI of a subject who underwent 25 CEMRI scans and 5400-cGy RT for pilocytic astrocytoma originating from the optic nerve. DN was unremarkable on the CT scan (not shown here) of this particular case. Initial HDN (A) becomes more obvious on follow-up studies performed $5(B)$ and $8(C)$ years after the first study with positive findings. Images $A$ and $B$ were obtained from 1.5T and $C$ was obtain from 3T scanners. This individual underwent 12 CEMRIs during the follow-up.

of contrast-enhanced studies was associated with a 2.92-fold odds of having HDN $(P<.001)$. The adjusted odds of HDN were estimated to be 12.3 times higher in subjects who underwent $>20$ enhanced MR imaging scans compared with patients undergoing $\leq 20$ scans (95\% CI, 4.1-37.0).

The rank sum test was used to investigate the difference between the total volume of gadolinium administration in the HDN and NDN groups. In the HDN group, the average total amount of gadolinium administration was significantly higher than that of NDN group (NDN group: median (p25 to p75), $40.0 \mathrm{~mL}$ (20.083.0); HDN group: median (p25 to p75), $76.0 \mathrm{~mL}$ (31.5-120.0); $P$ value $=.032$ ). Accordingly, the average total number of CEMRI scans before HDN become evident was significantly higher than the total number of CEMRI scans in the NDN group (NDN group: median [p25 to p75], 2.0 [1.0-5.0]; HDN group: median [p25 to p75], 5.0 [2.0-8.0]; $P$ value $=.004)$. We performed logistic regression with the presence of $\mathrm{HDN}$ as the dependent variable and ( 1 at a time) the log-transformed number of CEMRIs or the log-transformed total gadolinium volume as predictor variables. Every doubling of the number of CEMRIs was associated with 1.51 times higher odds of HDN (95\% CI, $1.14-2.01 ; P=.004)$. Every doubling of the total gadolinium volume was associated with 1.33 times higher odds of HDN (95\% CI, 1.02-1.73; $P=.038$ ). In multiple logistic regression analyses when both (log-transformed number of CEMRIs and log-transformed total gadolinium volume) were added as predictors, the log-transformed number of CEMRI scans remained statistically significant $(P=.016)$. The diagnostic efficiency of the number of CEMRIs and total gadolinium dose was determined by using receiver operating characteristic analysis. After $\geq 4$ CEMRI scans $(n=137$, ROC area $=0.642$, standard error $=0.046$, [95\% CI, 0.556-0.722]) or a total dose of $77 \mathrm{~mL}$ of gadolinium ( $n=137$ receiver operating characteristic area $=0.6076$, standard error $=0.0485$, [95\% CI, 0.5190.688 ), there was a significant increase in the likelihood of developing HDN. The best averages of sensitivity and specificity were found at the thresholds of 4 and 6 CEMRIs, with the threshold of 4 having higher sensitivity but lower specificity (sensitivity, $62.5 \%$; specificity, $61.4 \%$ ) and the threshold of 6 having lower sensitivity but higher specificity (sensitivity, 46.3\%; specificity, $77.2 \%)$. In sensitivity analyses, we also stratified analyses by younger than 18 years of age and equal to or older than 18 years. The point estimate of the association of the number of CEMRI studies with HDN was similar in both children and adults. However, because of the small sample size of children $(n=20)$, this did not reach statistical significance $(P=.11)$.

\section{Follow-Up Impact}

The average time interval between completion of RT and the first detection of HDN was 63 months (median [p25 to p75], 54 months [3.0-54.0 months], range, 3-324 months). The average radiologic follow-up time was 62.5 months for all subjects (median [p25 to p75] for NDN, 14 months [5.5-48.5 months]; and for HDN, 82 months [41.0-109.0 months]; range, 1-356 months, $P<.001)$. For those with HDN, the mean follow-up time after the first study with positive findings was 35 months (median [p25 to p75], 23 months [9.0-60.0 months]; range, 1-139 months). Twelve cases did not have follow-up studies after the first month of detection of HDN. On follow-up, 2 cases (2.2\%) reverted to a normal DN signal after being bright (after 5 and 36 months). All cases with HDN had bilateral hyperintensity initially. However, in 2 cases, the hyperintensity of $1 \mathrm{DN}$ disappeared and left only a unilateral HDN at the 122nd and 134th months of follow-up. The longest follow-up of a patient who developed HDN that persisted was 11.5 years after initial detection (Fig 2).

\section{Impact of Diagnosis Relates to Number of CEMRIs and Follow-Up Period}

Various pathologic diagnoses in both the NDN and HDN groups had a distinct distribution (Table 2).The incidence of World Health Organization grades III and IV malignant tumors in the group with NDN was higher than that of the group with HDN, and this difference was statistically significant $(P<.001)$. Sixtyone percent of patients were diagnosed with glioblastoma multiforme in the group with NDN, whereas this rate was only $16 \%$ in the group with HDN. Eighty percent of the patients in the group negative for HDN died during the follow-up as opposed to $58 \%$ mortality in the HDN group $(P=.002)$.

\section{No Impact of Demographic Variables}

Race was classified as white versus nonwhite because of the lower representation of some races. The race of 146 subjects was white, while 38 subjects were nonwhite. The nonwhite group included 23 African Americans, 2 Asians, 2 Hispanics, and 11 other (multiracial) subjects. The race and age demographic between the $\mathrm{NDN}$ and HDN groups was not statistically significant $(P=.4)$. 
Table 2: Diagnoses of brain tumors in the group with NDN and those with $\mathrm{HDN}^{\mathrm{a}}$

\begin{tabular}{lcc}
\hline \multicolumn{1}{c}{ Histology } & $\begin{array}{c}\text { NDN } \\
(\boldsymbol{n}=81)(\%)\end{array}$ & $\begin{array}{c}\text { HDN } \\
(\boldsymbol{n}=103)(\%)\end{array}$ \\
\hline Anaplastic astrocytoma & $8(9.9)$ & $19(18.4)$ \\
Astroblastoma & $0(0.0)$ & $1(1.0)$ \\
Astrocytoma & $4(4.9)$ & $14(13.6)$ \\
Astrocytoma, pilocytic & $2(2.5)$ & $4(3.9)$ \\
Desmoplastic nodular & $0(0.0)$ & $1(1.0)$ \\
$\quad$ medulloblastoma & & \\
Ependymoma & $4(4.9)$ & $5(4.9)$ \\
Fibrillary astrocytoma & $1(1.2)$ & $4(3.9)$ \\
Gemistocytic astrocytoma & $1(1.2)$ & $0(0.0)$ \\
Giant cell glioblastoma & $1(1.2)$ & $0(0.0)$ \\
Glioblastoma & $50(61.7)$ & $16(15.5)$ \\
Glioma, malignant & $4(4.9)$ & $7(6.8)$ \\
Medulloblastoma & $3(3.7)$ & $7(6.8)$ \\
Medullomyoblastoma & $0(0.0)$ & $1(1.0)$ \\
Mixed glioma & $0(0.0)$ & $3(2.9)$ \\
Myxopapillary ependymoma & $0(0.0)$ & $2(1.9)$ \\
Oligodendroglioma & $2(2.5)$ & $10(9.7)$ \\
Oligodendroglioma, anaplastic & $1(1.2)$ & $7(6.8)$ \\
Pleomorphic xanthoastrocytoma & $0(0.0)$ & $1(1.0)$ \\
Primitive neuroectodermal tumor & $0(0.0)$ & $1(1.0)$ \\
\hline
\end{tabular}

${ }^{a}$ Note the high percentages of glioblastoma multiforme in the NDN group.

Statistical analyses showed no relationship between HDN and variables such as sex, radiation site, creatinine levels, calcium/ phosphate levels, and history of chemotherapy. Of all included cases, $84(45.6 \%)$ subjects received chemotherapy, and there was no significant difference between the NDN and HDN groups in terms of a history of chemotherapy $(46.9 \%$ for the NDN and $44.6 \%$ for the HDN group).

\section{No Impact of MFS}

The possible impact of MFS was evaluated by interpreting repeat studies on different MFSs when available. The total number of MR images investigated was 733 . The MFS was $1.5 \mathrm{~T}$ for 690 studies and $3 \mathrm{~T}$ in the remaining 43 studies. The effect of the MFS was analyzed by the following: whether any $3 \mathrm{~T}$ scan was performed, by the number of 3T scans performed, and by the percentage of 3T scans performed per individual. There was no statistically significant difference between the HDN and NDN groups for all 3 parameters $(P=$ $.46, .50$, and .36 , respectively). One reader also qualitatively evaluated whether the MFS changed his final assessment of DN intensity in serial MR images with both $1.5 \mathrm{~T}$ and $3 \mathrm{~T}$ magnets. The MFS did not change the reader's final decision in any of the cases.

\section{Impact of Gadolinium Agent Used}

For further reliable analyses of gadolinium agents, we excluded cases that had at least 1 outside MR image and those lacking technique and dosage information in at least 1 CEMRI scan during the follow-up period ( $n=47)$. One hundred thirty-seven (female, 54 ; male, 83) cases were therefore included in our core subset analysis. In 113 of 137 individuals who were included for subset analysis, every CEMRI study was performed using a Magnevist (gadopentetate dimeglumine) injection. In all except 1 of the remaining cases $(n=23)$, at least 1 different gadolinium molecule including Magnevist was injected during the follow-up (Table 3). Given the preponderance of Magnevist administration, we collapsed the group with "any kind of non-Magnevist usage " during the fol-
Table 3: Number of CEMRI scans, total gadolinium doses, and commercial agents one-by-one in the group with HDN and NDN with doses collapsed

\begin{tabular}{|c|c|c|}
\hline Contrast Medium & NDN $(n=57)$ & $\operatorname{HDN}(n=80)$ \\
\hline $\begin{array}{l}\text { No. of CEMRIs (p25 to } \\
\text { p75) (median) }\end{array}$ & $2.0(1.0-5.0), 57$ & $5.0(2.0-8.0), 80$ \\
\hline $\begin{array}{l}\text { Total gadolinium } \\
\qquad \text { (p25 to p75) (median) }\end{array}$ & 40.0 (20.0-83.0), 57 & 76.0 (31.5-120.0), 80 \\
\hline Magnevist & $51(89.5)$ & $62(77.5)$ \\
\hline \multicolumn{3}{|l|}{$\begin{array}{l}\text { Any non-Magnevist } \\
\text { agent used }\end{array}$} \\
\hline No & $51(89.5)$ & $62(77.5)$ \\
\hline Yes & $6(10.5)$ & $18(22.5)$ \\
\hline \multicolumn{3}{|l|}{ Omniscan } \\
\hline No & $53(93.0)$ & $70(87.5)$ \\
\hline Yes & $4(7.0)$ & $10(12.5)$ \\
\hline \multicolumn{3}{|l|}{ MultiHance } \\
\hline No & $53(93.0)$ & $75(93.8)$ \\
\hline Yes & $4(7.0)$ & $5(6.3)$ \\
\hline \multicolumn{3}{|l|}{ ProHance } \\
\hline No & $57(100.0)$ & 78 (97.5) \\
\hline Yes & $0(0.0)$ & $2(2.5)$ \\
\hline \multicolumn{3}{|l|}{ OptiMARK } \\
\hline No & $57(100.0)$ & $79(98.8)$ \\
\hline Yes & $0(0.0)$ & $1(1.3)$ \\
\hline \multicolumn{3}{|l|}{ Gadavist } \\
\hline No & $57(100.0)$ & $79(98.8)$ \\
\hline Yes & $0(0.0)$ & $1(1.3)$ \\
\hline
\end{tabular}

low-up into a single category. The Fisher exact test showed no statistically significant difference between Magnevist and nonMagnevist usage $(P=.109)$.

\section{No Impact of $R T$}

Data records of total RDs were available for 129 (NDN: $n=58$; HDN: $n=71)$ subjects. Of this group, specific RD exposures of the DN were retrieved for 109 subjects (NDN: $n=52$; HDN: $n=$ 57). Total RT duration and fraction data were available for 108 subjects (NDN: $n=52$; HDN: $n=56$ ). The median value of total $\mathrm{RD}$ exposure of the brain was higher in the NDN group than in the HDN group (median [p25 to p75] for NDN, 5940.0 cGy [5375.0$6000.0 \mathrm{cGy}$ ]; for the HDN group: $5400.0 \mathrm{cGy}$ [5040.0-5940.0 cGy], $P=.017$ ). The DN was located outside the outermost circles (indicating the lowest radiation exposure) on all 3 imaging planes in 23 subjects with NDN and 25 subjects with HDN. Those cases were considered to have received a negligible amount of radiation exposure and were censored in the statistical analysis because $\mathrm{RD}$ could not be numerically quantified when the $\mathrm{DN}$ was located outside the $\mathrm{RF}$ (ie, the lowest circle). In cases with DN located within the colored isodose lines (ie, within the RF), the mean RDs were 3181 and 3261 cGy, respectively, for the groups with NDN and HDN (NDN: $n=29$; HDN: $n=32$ ). The mean number of fractions during RT was 31 (NDN) and 29 (HDN) days for the 2 groups. There was no statistically significant difference between 2 groups (those with NDN and $\mathrm{HDN}$ ) on the $\chi^{2}$ test for total $\mathrm{RD}$, specific $\mathrm{RD}$, and therapy duration $(P=.12, .86$, and .30 , respectively).

\section{No Confounders of Calcification or Hemorrhage}

The CT studies of 44 of the subjects with HDN who had CTs performed were unremarkable in terms of the appearance of the DN. Calcification and hemorrhage, common causes of T1WI hyperintensity, were excluded by these CT scans. 


\section{No HDN in Control Group}

The healthy control group underwent MR imaging without contrast agent administration. None of the control group had HDN. In quantitative signal-intensity analyses, the median (p25 to p75) of the ROI value was 380.4 (354.4-407.5) for average DN intensity and 384.7 (364.9-409.9) for average middle cerebellar peduncle intensity. The rank sum test for the difference between the 2 did not reach statistical significance $(P=.46)$.

\section{DISCUSSION}

In this study, analyses of multiple longitudinal MR images with long follow-up periods revealed that once HDN was evident, it was most likely to remain permanently, as long as 139 months after onset. Among various variables we investigated, our data suggest a statistically significant association between HDN and repeat CEMRI scans in patients who underwent various amounts of brain irradiation. An increase in the total number of CEMRI scans and thus total amount of gadolinium administration significantly increased the risk for developing HDN. After $\geq 4$ CEMRI scans and a total dose of $77 \mathrm{~mL}$ of gadolinium, there was a significant increase in the likelihood of developing HDN. No significant association was found between doses of radiation exposure to DN and HDN on T1WI. Although the radiation dosing schema used differed in potential biologic impact because the total dose and number and size of fractions varied by diagnosis and tumor size, no relationship was encountered between whether a significant dose was given to the dentate nucleus and HDN. While it cannot be excluded that a subgroup of patients treated with a particular regimen might be vulnerable to such an effect, the absence of any observed association suggests that this is not the case. We also showed that the number of the subjects whose DNs were located outside the outermost RF did not significantly differ between subjects with HDN and those with NDN. Thus, the DNs do not necessarily need to be within the RF to become hyperintense on T1WI.

Gadolinium, a molecule known to induce cell damage, has been shown to deposit in normal and pathologic human tissue in different studies. ${ }^{9-12}$ There is evidence that in individuals with a history of multiple CEMRI scans, gadolinium-based MR imaging contrast agents can deposit for up to 3 years in structures such as kidney, skin, muscle, and bone after becoming insoluble free gadolinium $^{+3}$ as a result of transmetalation and dissociation processes. ${ }^{13}$ We have verified that HDN on T1WI possibly results from gadolinium deposits that can persist longer than 11 years. Different gadolinium molecules have different kinetics and thus different propensities for dechelation. ${ }^{14}$ Omniscan (gadodiamide) and Magnevist (gadopentetate dimeglumine) were used in the very first studies in the literature conducted on subjects with HDN on T1WI. ${ }^{7,8}$ In the present retrospective study, we primarily used Magnevist (gadopentetate dimeglumine) for CEMRI scans in subjects with normal renal function during the time of this study. Statistical analyses yielded no significant association between serum creatinine level and the development of HDN. Omniscan (gadodiamide), MultiHance (gadobenate dimeglumine), ProHance (gadoteridol), OptiMARK (gadoversetamide), and Gadavist (gadobutrol) were also used in some cases. The sample sizes of other commercial gadolinium agents were too small compared with Magnevist to make a reliable sta- tistical analysis for individual gadolinium agents. Nevertheless, there was no statistically significant difference between the HDN incidences by using Magnevist and a combination of different gadolinium agents. We recommend further evaluation.

In the present study, we investigated the associations between an HDN and the components of RT such as total RD to brain, specific RD to the DN, and the number of fractions during RT. No statistically significant association was found between any of these variables and HDN on T1WI. These findings were contrary to what was suggested by Kasahara et al, ${ }^{7}$ who found a correlation between the brightness of the $\mathrm{DN}$ and the amount of radiation exposure. In terms of underlying brain pathologies, we found a significant difference in dispersion of histopathologic diagnoses of brain neoplasms between the group with NDN and that with HDN. Malignant tumors with low survival rates such as glioblastoma multiforme were more likely to be in the NDN group but also underwent fewer enhanced scans because of that shorter life span. During the follow-up period, the death rate was significantly higher in the NDN group compared with the HDN group. We speculate that the early death of subjects with high-grade tumors led to a decreased total number of follow-up MR imaging studies, thus the total amount of gadolinium exposure and a lower rate of HDN.

Hyperintensity on T1WI in the basal ganglia has been shown to be caused by various entities. Total parenteral nutrition supplemented with manganese has proved to cause abnormal high signal intensity at the globus pallidus on T1WI. ${ }^{15-17}$ It has been speculated that a hyperintense globus pallidus in patients with liver failure and significant liver collaterals is also related to the manganese accumulation in the basal ganglia by virtue of bypass conduits that skip the hepatic clearance. ${ }^{18}$ In light of these studies, we excluded patients on total parenteral nutrition therapy and those with liver failure. Iron is one of the best-known causes of T1 shortening on MR imaging along with several other substances. ${ }^{15-19}$ Calcium deposition in cortical and deep gray matter has also been associated with paradoxical T1 shortening on MR imaging. ${ }^{20}$ None of our patients had a history of iron metabolism disorder. We did not find a statistically significant relationship between serum calcium or phosphate levels and HDN on T1WI. $\mathrm{RT}$ is reported to cause calcification in brain tissue. ${ }^{2,3}$ However, there was no radiologic evidence of DN calcium accumulation on CT examinations of subjects included in our study.

Prior studies showed that the MFS may contribute to contrast differences between brain structures, which could have affected the appreciation of signal alteration. ${ }^{21}$ In the present study, we found no significant difference among the scanners with different MFSs in qualitatively evaluating the appearances of DN on T1WI. There was also no statistically significant difference between scanner strengths (1.5T and 3T) in terms of HDN incidence. Most of our patients underwent CEMRI with a $1.5 \mathrm{~T}$ scanner because the present study extended back to 1995 .

In the English literature, few studies have investigated HDN on T1WI, and each drew different conclusions for possible etiologies. ${ }^{5-8}$ Roccaglita et $\mathrm{al}^{8}$ reported a higher incidence of HDN on T1WI in a subset of patients with secondary-progressive MS compared with 2 other groups comprising patients with relapsingremitting and primary-progressive MS subtypes. In a study conducted on 362 adult patients, Kasahara et $\mathrm{al}^{7}$ considered the 
relationship between $\mathrm{T} 1$ hyperintensiy in $\mathrm{DN}$ and variables such as brain irradiation, multiple sclerosis, and liver dysfunction. They found a significant association between brain irradiation history and T1 hyperintensiy in DN. However, details about interaction or correlation between whole- and partial-brain irradiation, if any, were not discussed in these studies, and they did not look at the number of gadolinium-enhanced MR imaging examinations as a variable. Kanda et $\mathrm{al}^{6}$ compared subjects who underwent at least 6 CEMRI scans with subjects who underwent at least 6 unenhanced MR imaging scans and found a significant association between T1 hyperintensity of GP and DN and the total number of CEMRIs. Most recently Errante et $\mathrm{al}^{5}$ replicated the same technique with Kanda et al, excluding the globus pallidus, and reinforced the findings of Kanda et al. They supported the idea of a strong association of the total number of gadolinium administrations with increased T1 shortening in the human DN by showing a linear regression model in cases with repeat CEMRI scans in their retrospective study. ${ }^{7}$

The lack of a histologic examination and thus a molecular investigation of DN was the main limitation of our observational study. Although in receiver operating characteristic analyses, 4 CEMRI scans were significantly associated with a higher percentage of HDN, we did not detect an absolute threshold for the number of CEMRI scans resulting in HDN. Magnevist was administrated in all scans in a vast majority of our subjects. A combination of different agents was used during the follow-up scans in the remainder of the subjects. Therefore, further studies with evenly distributed commercial gadolinium molecules are needed to reliably compare the association of HDN and other gadolinium molecules. Given the retrospective design of our study, the time intervals between studies and the time interval between the last day of RT and the first MR imaging scan and/or first detection of HDN could not be adjusted. This situation resulted in different follow-up times and thus variable timing of the detection of HDN. Because the follow-up period was longer than a decade for some instances, body mass index was not evaluated to prevent the potential interactions of body weight fluctuations in run-time. Because this was a retrospective, observational, radiologic study, we did not seek the clinical consequences of HDN. Our goal was to elucidate an imaging finding, not its clinical significance.

\section{CONCLUSIONS}

Our study revealed that repeat performance of CEMRI studies in irradiated subjects contributes to a hyperintense appearance of the DN on T1WI. This appearance may be related to the accumulation of gadolinium in the DN. Other variables that could be associated with HDN demonstrated no significant effect on the DN appearance in this retrospective study.

Disclosures: Dhananjay Vaidya—UNRELATED: Consultancy: Consumable Science, Comments: included consultancy regarding the filing of a pending patent; Grants/ Grants Pending: National Institutes of Health.* David M. Yousem-UNRELATED: Expert Testimony: consultations on medicolegal cases; Payment for Lectures (including service on Speakers Bureaus): American College of Radiology Education Center*; Royalties: Elsevier for 3 books; Payment for Development of Educational Presentations: CMElnfo.com for Continuing Medical Education.* *Money paid to the institution.

\section{REFERENCES}

1. Jain R, Robertson PL, Gandhi D, et al. Radiation-induced cavernomas of the brain. AJNR Am J Neuroradiol 2005;26:1158-62

2. Lee KF, Suh JH. CT evidence of grey matter calcification secondary to radiation therapy. Comput Tomogr 1977;1:103-10

3. Valk PE, Dillon WP. Radiation injury of the brain. AJNR Am J Neuroradiol 1991;12:45-62

4. Perreault S, Lober RM, Cheshier S, et al. Time-dependent structural changes of the dentatothalamic pathway in children treated for posterior fossa tumor. AJNR Am J Neuroradiol 2014;35:803-07

5. Errante Y, Cirimele V, Mallio CA, et al. Progressive increase of T1 signal intensity of the dentate nucleus on unenhanced magnetic resonance images is associated with cumulative doses of intravenously administered gadodiamide in patients with normal renal function, suggesting dechelation. Invest Radiol 2014;49:685-90

6. Kanda T, Ishii $\mathrm{K}$, Kawaguchi $\mathrm{H}$, et al. High signal intensity in the dentate nucleus and globus pallidus on unenhanced T1-weighted MR images: relationship with increasing cumulative dose of a gadolinium-based contrast material. Radiology 2014;270:834-41

7. Kasahara S, Miki Y, Kanagaki M, et al. Hyperintense dentate nucleus on unenhanced T1-weighted MR images is associated with a history of brain irradiation. Radiology 2011;258:222-28

8. Roccatagliata L, Vuolo L, Bonzano L, et al. Multiple sclerosis: hyperintense dentate nucleus on unenhanced T1-weighted MR images is associated with the secondary progressive subtype. Radiology 2009;251:503-10

9. Zhao J, Zhou ZQ, Jin JC, et al. Mitochondrial dysfunction induced by different concentrations of gadolinium ion. Chemosphere 2014;100:194-99

10. Ray DE, Cavanagh JB, Nolan CC, et al. Neurotoxic effects of gadopentetate dimeglumine: behavioral disturbance and morphology after intracerebroventricular injection in rats. AJNR Am J Neuroradiol 1996;17:365-73

11. Abraham JL, Thakral C. Tissue distribution and kinetics of gadolinium and nephrogenic systemic fibrosis. Eur J Radiol 2008;66:200-07

12. Thakral C, Abraham JL. Nephrogenic systemic fibrosis: histology and gadolinium detection. Radiol Clin North Am 2009;47:841-53, vi-vii

13. Thakral C, Alhariri J, Abraham JL. Long-term retention of gadolinium in tissues from nephrogenic systemic fibrosis patient after multiple gadolinium-enhanced MRI scans: case report and implications. Contrast Media Mol Imaging 2007;2:199-205

14. Sieber MA, Lengsfeld P, Frenzel T, et al. Preclinical investigation to compare different gadolinium-based contrast agents regarding their propensity to release gadolinium in vivo and to trigger nephrogenic systemic fibrosis-like lesions. Eur Radiol 2008;18:2164-73

15. Boyko OB, Burger PC, Shelburne JD, et al. Non-heme mechanisms for T1 shortening: pathologic, CT, and MR elucidation. AJNR Am J Neuroradiol 1992;13:1439-45

16. Mirowitz SA, Sartor K, Gado M. High-intensity basal ganglia lesions on T1-weighted MR images in neurofibromatosis. AJR Am J Roentgenol 1990;154:369-73

17. Moore DF, Ye F, Schiffmann R, et al. Increased signal intensity in the pulvinar on T1-weighted images: a pathognomonic MR imaging sign of Fabry disease. AJNR Am J Neuroradiol 2003;24:1096-101

18. Inoue E, Hori S, Narumi Y, et al. Portal-systemic encephalopathy: presence of basal ganglia lesions with high signal intensity on MR images. Radiology 1991;179:551-55

19. Vymazal J, Righini A, Brooks RA, et al. T1 and T2 in the brain of healthy subjects, patients with Parkinson disease, and patients with multiple system atrophy: relation to iron content. Radiology 1999;211:489-95

20. Suzuki S, Nishio S, Takata K, et al. Radiation-induced brain calcification: paradoxical high signal intensity in T1-weighted MR images. Acta Neurochir (Wien) 2000;142:801-04

21. Fushimi Y, Miki Y, Urayama S, et al. Gray matter-white matter contrast on spin-echo $\mathrm{T} 1$-weighted images at $3 \mathrm{~T}$ and $1.5 \mathrm{~T}$ : a quantitative comparison study. Eur Radiol 2007;17:2921-25 